\title{
Very large magnetoresistance and spin state transition in Ba-doped cobaltites
}

\author{
I. O. Troyanchuk, ${ }^{1, a)}$ M. V. Bushinsky, ${ }^{1}$ A. V. Nikitin, ${ }^{1}$ L. S. Lobanovsky, ${ }^{1}$ A. M. Balagurov, ${ }^{2}$ \\ V. Sikolenko, ${ }^{2,3}$ V. Efimov, ${ }^{2}$ and D. V. Sheptyakov ${ }^{4}$ \\ ${ }^{1}$ SSPA “Scientific-Practical Materials Research Centre of NAS of Belarus," P. Brovka str. 19, 220072 Minsk, \\ Belarus \\ ${ }^{2}$ Joint Institute for Nuclear Research, Dubna RU-141980, Russia \\ ${ }^{3}$ Karlsruhe Institute of Technology, DE-76131 Karlsruhe, Germany \\ ${ }^{4}$ Laboratory for Neutron Scattering, Paul Scherrer Institut, 5232 Villigen PSI, Switzerland
}

(Received 13 November 2012; accepted 17 January 2013; published online 6 February 2013)

\begin{abstract}
Structural, magnetization, and magnetotransport measurements have been performed for anion deficient $\mathrm{La}_{0.5} \mathrm{Ba}_{0.5} \mathrm{Co}_{1-x} \mathrm{Fe}_{x} \mathrm{O}_{3-\delta}(x \leq 0.4)$ and $\mathrm{La}_{1-y} \mathrm{Ba}_{y} \mathrm{CoO}_{3-\delta}(0.5 \leq y \leq 0.6)$ perovskites. It has been found that the iron doped compositions with $x \leq 0.23$ are predominantly ferromagnetic. The Curie point and magnetization are slightly larger in insulating phase $(x=0.1)$ than in metallic one $(x=0.05)$. Magnetoresistance ratio rises with lowering temperature and increasing iron content reaching three orders of magnitude in predominantly antiferromagnetic state $(x \geq 0.25)$. The rise of the barium content in $\mathrm{La}_{1-y} \mathrm{Ba}_{y} \mathrm{CoO}_{3-\delta}$ series above $y=0.5$ leads to stabilization of antiferromagnetic phase and strong enhancement of the magnetoresistance. Antiferromagnetic ordering is accompanied by increase in resistivity. The lack of the low field intergrain magnetoresistance is in agreement with a weak spin polarization of the charge carriers. Magnetoresistance is associated with antiferromagnet-ferromagnet (from mixed high/low into intermediate spin state) transition induced by magnetic field. It is suggested that ferromagnetism is originated from superexchange interaction via oxygen. (C) 2013 American Institute of Physics. [http://dx.doi.org/10.1063/1.4790316]
\end{abstract}

\section{INTRODUCTION}

Cobaltites with perovskite-like structure attract much interest as they exhibit a variety of phase transitions due to realization of different spin states of Co ions and strong correlation between magnetic and transport properties. The parent $\mathrm{LaCoO}_{3-\delta}$ is mostly in low spin state at low temperatures. ${ }^{1-5}$ As temperature increases, a gradual transition to insulating paramagnetic state occurs due to excitation of $\mathrm{Co}^{3+}$ ions from low spin to intermediate or high spin state. At temperatures above $530 \mathrm{~K}$, a metallic state appears. It was suggested that the spin transition is assisted by defects ${ }^{4}$ and goes through phase separated state. ${ }^{5}$ The spin transition and metallic state can be obtained as well by substitution of La ions for alkaline earth ones. ${ }^{1-7}$ For example, in system $\mathrm{La}_{1-y} \mathrm{Sr}_{y} \mathrm{CoO}_{3-\delta}$ near $y \approx 0.18$ the metallic ferromagnetic state is established whereas at lower strontium content a spinglass behavior is observed. ${ }^{6}$ However, upon barium doping the spin glass at $y<0.16$ transforms into the antiferromagnetic long-range order $(y \sim 0.17)$ passing gradually to the insulating ferromagnetic state through the two-phase region $(0.17<y<0.20)$, and then to the metallic ferromagnetic one $(y \approx 0.22)$. Remarkable that the magnetic field strongly suppresses an antiferromagnetic phase. ${ }^{8}$ Unfortunately, the oxygen nonstoichiometry has not been reported. ${ }^{7-9}$

The "stoichiometric" $\mathrm{La}_{0.5} \mathrm{Ba}_{0.5} \mathrm{CoO}_{3}$ and $\mathrm{La}_{0.5} \mathrm{Sr}_{0.5} \mathrm{CoO}_{3-\delta}$ compositions exhibit different electrical conductivity and

\footnotetext{
a) Author to whom correspondence should be addressed. Electronic mail: troyan@physics.by.
}

magnetoresistance in spite of similar magnetic properties. Both these compounds are ferromagnets with $\mathrm{T}_{\mathrm{C}} \sim 180$ and $250 \mathrm{~K}$, respectively. It has been suggested that $\mathrm{Co}^{3+}$ and $\mathrm{Co}^{4+}$ ions are in the intermediate spin state being Jahn-Teller active. ${ }^{10} \mathrm{La}_{0.5} \mathrm{Sr}_{0.5} \mathrm{CoO}_{3-\delta}$ exhibits a metallic behavior in both paramagnetic and ferromagnetic phases. However, the "stoichiometric" $\mathrm{La}_{0.5} \mathrm{Ba}_{0.5} \mathrm{CoO}_{3}$ gradually transforms from metallic into a weakly pronounced insulating state below $\mathrm{T} \sim 140 \mathrm{~K} .^{10,11}$ Metal-insulator transition is accompanied with a tetragonal structural distortion interpreted as indication of static Jahn-Teller ordering of $e_{g}$ orbitals of Co ions. ${ }^{11}$ $\mathrm{La}_{0.5} \mathrm{Ba}_{0.5} \mathrm{CoO}_{3}$ exhibits a large magnetoresistive effect at low temperature, ${ }^{10,11}$ whereas in metallic $\mathrm{La}_{0.5} \mathrm{Sr}_{0.5} \mathrm{CoO}_{3-\delta}$ magnetoresistance is very small. ${ }^{12,13}$ An increasing of the oxygen vacancies in $\mathrm{La}_{0.5} \mathrm{Ba}_{0.5} \mathrm{CoO}_{3-\delta}$ causes insulating behavior and macroscopic structural phase separation into antiferromagnetic and ferromagnetic phases with different cobalt spin states. ${ }^{13}$

The Ti doping of $\mathrm{La}_{0.5} \mathrm{Ba}_{0.5} \mathrm{CoO}_{3-\delta}$ leads to appearance of a well pronounced peak in magnetization-temperature curves. ${ }^{14}$ The peak of magnetization has been ascribed to antiferromagnetism. The applied pressure stabilizes the ferromagnetic phase. The substitution of the cobalt by iron in $\mathrm{Pr}_{0.5} \mathrm{Sr}_{0.5} \mathrm{Co}_{1-x} \mathrm{Fe}_{x} \mathrm{O}_{3}$ causes a transition from ferromagnetic into spin glass state and concomitant enhancement of the magnetoresistance. ${ }^{15}$ However, effect of the iron doping on magnetotransport properties of the $\mathrm{La}_{1-y} \mathrm{Ba}_{y} \mathrm{CoO}_{3-\delta}$ series is not studied yet.

The nature of the ferromagnetic state in cobaltites was a subject of debates in a long time. Three main mechanisms 
explaining magnetic properties of mixed-valence cobaltites were suggested: the superexchange model based on the localized electron interaction via oxygen ion, the Zener double exchange via charge transfer, and itinerant magnetism. ${ }^{16}$

In order to understand the origin of the large magnetoresistance and metal-insulator transition observed in stoichiometric $\mathrm{La}_{0.5} \mathrm{Ba}_{0.5} \mathrm{CoO}_{3-\delta}$, we have decided to study the heavily barium doped $\mathrm{La}_{1-y} \mathrm{Ba}_{y} \mathrm{CoO}_{3-\delta}(y>0.5)$ and iron doped $\mathrm{La}_{0.5} \mathrm{Ba}_{0.5} \mathrm{Co}_{1-x} \mathrm{Fe}_{x} \mathrm{O}_{3-\delta}$ compositions. It is found that both barium $(y>0.5)$ and iron doping lead to strong enhancement of the magnetoresistance and concomitant ferromagnet-antiferromagnet transition. A very large magnetoresistance is ascribed to antiferromagnet-ferromagnet transition induced by external magnetic field.

\section{EXPERIMENTAL}

Polycrystalline samples with compositions $\mathrm{La}_{1-y} \mathrm{Ba}_{y} \mathrm{CoO}_{3-\delta}$ $(0.5 \leq y \leq 0.6) \quad$ and $\quad \mathrm{La}_{0.5} \mathrm{Ba}_{0.5} \mathrm{Co}_{1-x} \mathrm{Fe}_{x} \mathrm{O}_{3-\delta} \quad(x \leq 0.4)$ have been prepared from oxides $\mathrm{La}_{2} \mathrm{O}_{3}$, previously dried at $1000{ }^{\circ} \mathrm{C}$ in air, $\mathrm{CoO}$ and $\mathrm{Fe}_{2} \mathrm{O}_{3}$ as well as carbonate $\mathrm{BaCO}_{3}$ of purity of not less than $99.9 \%$, mixed in stoichiometric ratio using a planetary ball mill RETSCH PM100. Preliminary annealing has been performed at $1000{ }^{\circ} \mathrm{C}$. The $\mathrm{La}_{1-y} \mathrm{Ba}_{y} \mathrm{CoO}_{3-\delta}$ samples were synthesized at $\mathrm{T}=1180^{\circ} \mathrm{C}$ and $\mathrm{T}=1130^{\circ} \mathrm{C}$ for $12 \mathrm{~h}$ in air. The $\mathrm{La}_{1-y} \mathrm{Ba}_{y} \mathrm{CoO}_{3-\delta}$ compounds prepared at $1130{ }^{\circ} \mathrm{C}$ were additionally annealed at $300^{\circ} \mathrm{C}$ in air for $48 \mathrm{~h}$. The compositions doped with iron have been obtained at $1200^{\circ} \mathrm{C}$. All the samples have been cooled at a rate $80^{\circ} \mathrm{C} / \mathrm{h}$. Xray diffraction analysis was performed using diffractometer DRON-3 M. For some compositions, neutron powder diffraction (NPD) data were collected on the high resolution powder diffractometer HRPT at SINQ (PSI, Switzerland). The data were acquired in the range of $10^{\circ}$ $<2 \theta<165^{\circ}$ using a step of 0.05 and wavelength $1.494 \AA$. The crystal structure refinement has been performed by the Rietvield method with FULLPROF software package. ${ }^{17}$ Magnetization and transport properties in magnetic fields up to $14 \mathrm{~T}$ have been measured by a Physical Properties Measurement System (Cryogenic Ltd).

\section{RESULTS}

\section{A. Crystal structure}

$\mathrm{X}$-ray diffraction structural studies performed at room temperature have shown that all the compositions $\mathrm{La}_{0.5} \mathrm{Ba}_{0.5} \mathrm{Co}_{1-x} \mathrm{Fe}_{x} \mathrm{O}_{3-\delta}(x \leq 0.4)$ can be refined in perovskite structure with cubic symmetry (space group $P m \overline{3} m$ ). For the undoped composition $(x=0)$, the unit cell parameter $(a=3.890 \AA)$ is bigger than that for stoichiometric $\mathrm{La}_{0.5} \mathrm{Ba}_{0.5} \mathrm{CoO}_{3}$ compound $(\sim 3.885 \AA) .{ }^{10,11}$ Unit cell volume slightly increases with increasing iron content and synthesis temperature. The observed increase of unit cell volume is probably associated not only with Co substitution by $\mathrm{Fe}$ but also with an insignificant increase of oxygen vacancy concentration under iron doping. It is known that ferrites with perovskite structure prepared in air can be characterized by certain deviation from oxygen stoichiometry. ${ }^{18} \mathrm{La}_{1-y} \mathrm{Ba}_{y} \mathrm{CoO}_{3-\delta}$

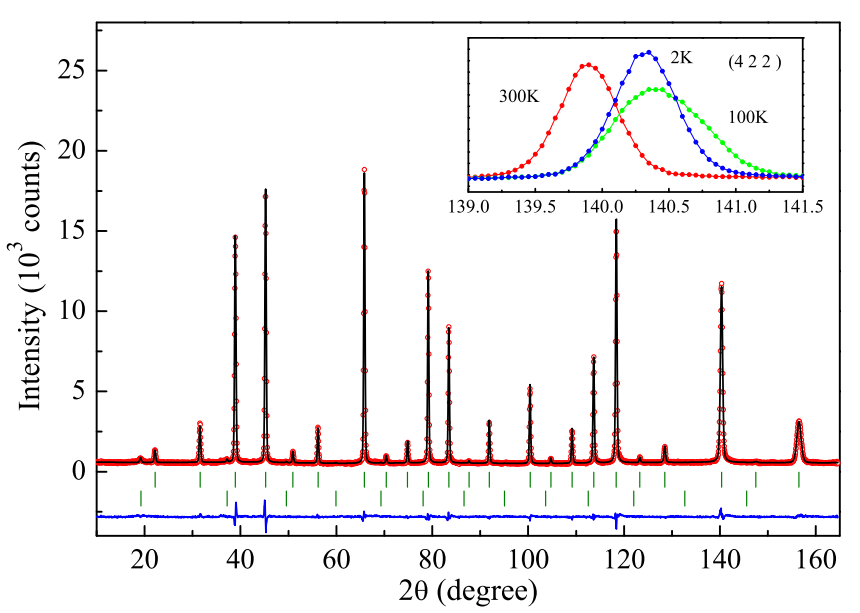

FIG. 1. Observed pattern, calculated profile, and difference curve of the Rietveld refinement done on the NPD data for the sample $\mathrm{La}_{0.5} \mathrm{Ba}_{0.5} \mathrm{Co}_{0.75} \mathrm{Fe}_{0.25} \mathrm{O}_{3-\delta}$ obtained at $2 \mathrm{~K}$. Ticks below the graph indicate the peak positions for nuclear and magnetic diffraction. The inset shows the zoomed in regions of the experimental data obtained at 2, 100, and $300 \mathrm{~K}$.

compositions with $0.5 \leq y \leq 0.6$ have cubic unit cell too. Unit cell parameter significantly increases with increasing $\mathrm{Ba}$ concentration.

NPD data for the $\mathrm{La}_{0.5} \mathrm{Ba}_{0.5} \mathrm{Co}_{0.75} \mathrm{Fe}_{0.25} \mathrm{O}_{3-\delta}$ and $\mathrm{La}_{0.44} \mathrm{Ba}_{0.56} \mathrm{CoO}_{3-\delta}$ compositions have been collected at $300 \mathrm{~K}, 100 \mathrm{~K}$, and $2 \mathrm{~K}$. Rietveld refinement carried out on the NPD patterns (Figs. 1 and 2) assumes cubic symmetry of the lattice (space group $P m \overline{3} m, a=3.8965 \AA$ and $3.9074 \AA$ correspondingly at room temperature). It should be noted though that in our refinements at any temperature, we observe a slight systematic mismatch between the experimental and ideal calculated peak positions for the cubic lattice. These inconsistencies are, however, so tiny that it does not allow for the search for any frozen-in symmetry distortion. This is also not the scope of the present study, and the agreement factors of the refinements are satisfactory. We did not observe obvious structure changes in either sample upon cooling down to $2 \mathrm{~K}$; however, diffraction peaks in the pattern of $\mathrm{La}_{0.5} \mathrm{Ba}_{0.5} \mathrm{Co}_{0.75} \mathrm{Fe}_{0.25} \mathrm{O}_{3-\delta}$ composition are significantly

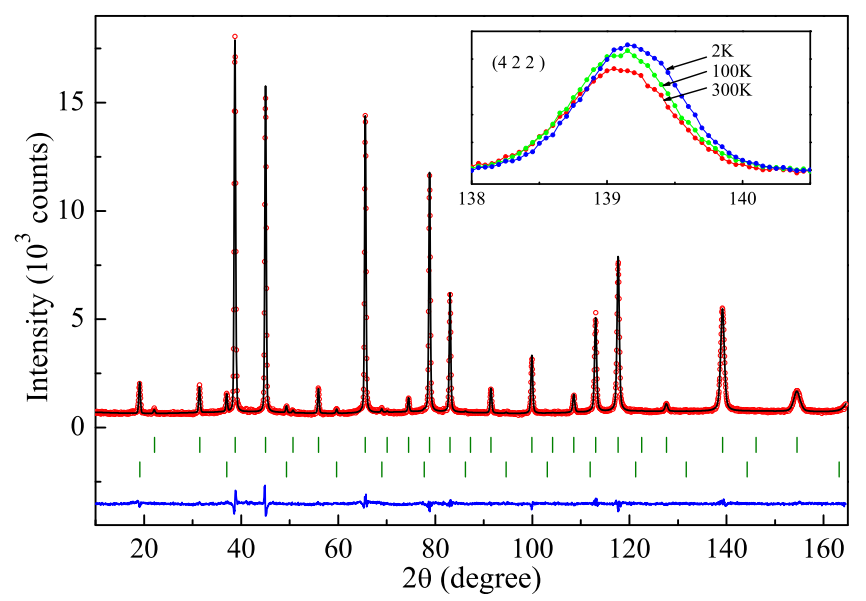

FIG. 2. Observed pattern, calculated profile, and difference curve of the Rietveld refinement done on the NPD data for the sample $\mathrm{La}_{0.44} \mathrm{Ba}_{0.56} \mathrm{CoO}_{3-\delta}$ obtained at $2 \mathrm{~K}$. Ticks below the graph indicate the peak positions for nuclear and magnetic diffraction. The inset shows the zoomed in regions of the experimental data obtained at 2, 100, and $300 \mathrm{~K}$. 
broadened at $100 \mathrm{~K}$ (see inset to Fig. 1). Microstructure analysis shows that the size-broadening effects remain negligible in the patterns of this composition at all the temperatures, providing an estimation of the volume-averaged coherent lengths of $\sim 2000 \AA$. Instead, it turns out that the microstress-related effects can be responsible for the peaks broadening at $100 \mathrm{~K}$. The average microstrain increases from $\sim 5 \times 10^{-4}$ at $300 \mathrm{~K}$ to $\sim 11 \times 10^{-4}$ at $100 \mathrm{~K}$, and then relaxes back to $\sim 6 \times 10^{-4}$ at $2 \mathrm{~K}$. However, the diffraction peaks shift forward to lower angles upon cooling from $100 \mathrm{~K}$ to $2 \mathrm{~K}$ (inset Fig. 1). Such a type of behavior could not been explained by relaxation process.

In the patterns of as prepared $\mathrm{La}_{0.44} \mathrm{Ba}_{0.56} \mathrm{CoO}_{3-\delta}$ composition, the microstructure effects at temperature around $100 \mathrm{~K}$ are not stable too (inset Fig. 2). Within the resolution limits of the medium resolution mode of HRPT, there are no size-related peak broadening effects observed, which puts the estimation for the lowest size of the coherently scattering domains in this material above $3000 \AA$. On the contrary, the microstress-related peak-broadening is strongly pronounced: average microstrain amounts to $\sim(1.6 / 1.7) \times 10^{-3}$ at room temperature. Refined oxygen stoichiometry at all three temperatures for both $\mathrm{La}_{0.5} \mathrm{Ba}_{0.5} \mathrm{Co}_{0.75} \mathrm{Fe}_{0.25} \mathrm{O}_{3-\delta}$ and $\mathrm{La}_{0.44} \mathrm{Ba}_{0.56} \mathrm{CoO}_{3-\delta}$ samples amounts to $2.93 \pm 0.03$.

Both $\mathrm{La}_{0.5} \mathrm{Ba}_{0.5} \mathrm{Co}_{0.75} \mathrm{Fe}_{0.25} \mathrm{O}_{3-\delta}$ and $\mathrm{La}_{0.44} \mathrm{Ba}_{0.56} \mathrm{CoO}_{3-\delta}$ compounds possess extremely stiff lattices. Upon cooling from 300 to $2 \mathrm{~K}$, their unit cell parameters only decrease very weakly: from 3.8965 to $3.8912 \AA$ for the former and only from 3.9074 to $3.9059 \AA$ for the latter which in this case provides an unprecedentedly low $300 \mathrm{~K}$-averaged linear thermal expansion coefficient: as low as $\sim 1.3 \times 10^{-6}$. In case of $\mathrm{La}_{0.5} \mathrm{Ba}_{0.5} \mathrm{Co}_{0.75} \mathrm{Fe}_{0.25} \mathrm{O}_{3-\delta}$, the refined unit cell constant at $2 \mathrm{~K}(3.8912 \AA)$ is even slightly bigger than at $100 \mathrm{~K}$ (3.8901 А).

In the both compounds, magnetic ordering manifests itself nicely in the NPD data at 100 and at $2 \mathrm{~K}$ (Figs. 1 and 2). Additional peaks in the NPD patterns of both compounds are indexed with the propagation vector $(1 / 2,1 / 2,1 / 2)$. Symmetry analysis carried out with the program SARAh ${ }^{19}$ and subsequent checks with the Rietveld refinements points uniquely to the G-type of magnetic ordering (AFM order between the spins of all the nearest neighbor ions). At the lowest temperature of $2 \mathrm{~K}$, the saturated magnetic moments per each site of $\mathrm{Co} / \mathrm{Fe}$ or Co exclusively amount only to 0.74(2) $\mu_{\mathrm{B}}$ in $\mathrm{La}_{0.5} \mathrm{Ba}_{0.5} \mathrm{Co}_{0.75} \mathrm{Fe}_{0.25} \mathrm{O}_{3-\delta}$, and 1.52(2) $\mu_{\mathrm{B}}$ in $\mathrm{La}_{0.44} \mathrm{Ba}_{0.56} \mathrm{CoO}_{3-\delta}$. The relatively small value of the magnetic moment can be associated with large spin glass-like magnetic component.

\section{B. Magnetic properties}

$\mathrm{La}_{0.5} \mathrm{Ba}_{0.5} \mathrm{CoO}_{3-\delta}$ has the Curie point about $170 \mathrm{~K}$ and magnetic moment $1.8 \mu_{\mathrm{B}}$ per cobalt ion. ${ }^{13}$ The temperature dependencies of magnetization measured upon cooling for the low doped compositions $\mathrm{La}_{0.5} \mathrm{Ba}_{0.5} \mathrm{Co}_{1-x} \mathrm{Fe}_{x} \mathrm{O}_{3-\delta}(0.05$ $\leq x \leq 0.25$ ) are presented in Fig. 3. One can see that substitution of $\mathrm{Co}$ ions for $\mathrm{Fe}$ ones results in some lowering of the Curie temperature in comparison with the undoped sample. The magnetic ordering takes place in the composition with

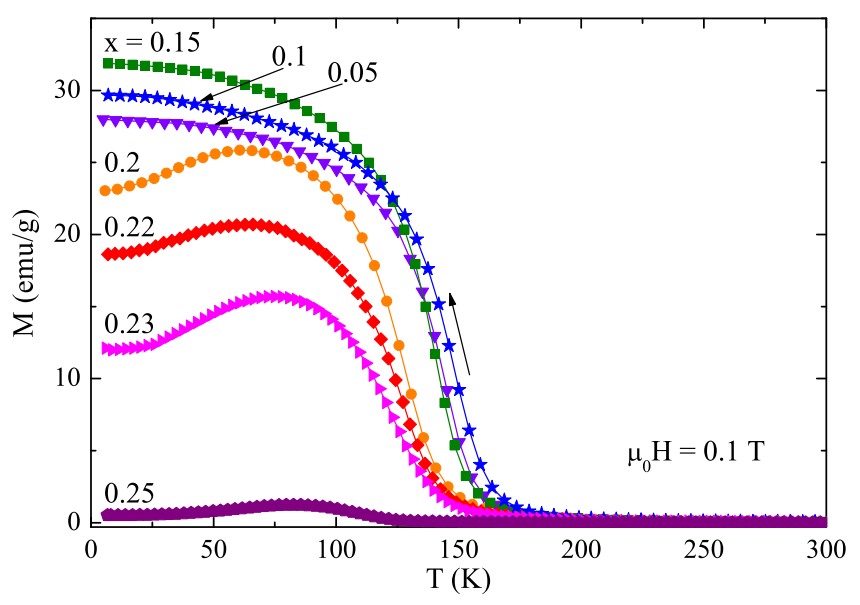

FIG. 3. Magnetization vs. temperature dependencies measured in a field of $0.1 \mathrm{~T}$ upon cooling.

$x=0.05$ near $147 \mathrm{~K}$, where the magnetization derivative on temperature has a maximal value. However, the Curie point of the $x=0.1$ sample is slightly larger than for $x=0.05$. Temperature dependencies of magnetization measured upon cooling in different magnetic fields for the compositions $x \geq$ 0.2 exhibit anomalous behavior: drop of the magnetization with temperature decreasing. The anomalous behavior of the magnetization is well pronounced for the $x=0.25$ (Fig. 3) and $x=0.3$ (Fig. 4) samples. Remarkable that the large difference between ZFC and FC magnetizations is persisted in a field of $14 \mathrm{~T}$ (Fig. 4). Such a behavior might indicate a ferromagnet-antiferromagnet transition and concomitant cobalt spin state transition induced by temperature lowering. This hypothesis is in agreement with the anomalous broadening of the diffraction peaks around $100 \mathrm{~K}$ (Fig. 1). The large magnetization revealed far above the Curie points can be associated with ferromagnetic clusters embedded in paramagnetic matrix. The maximum of the magnetization shifts to higher temperature and becomes much less pronounced with further iron content increasing.

Magnetization dependencies on magnetic field at $7 \mathrm{~K}$ are shown in Fig. 5. The compositions $x=0$ and 0.05 have approximately equal magnetization at $\mathrm{T}=7 \mathrm{~K}$. For the compositions $x=0.1$ and $x=0.15$, spontaneous magnetization is

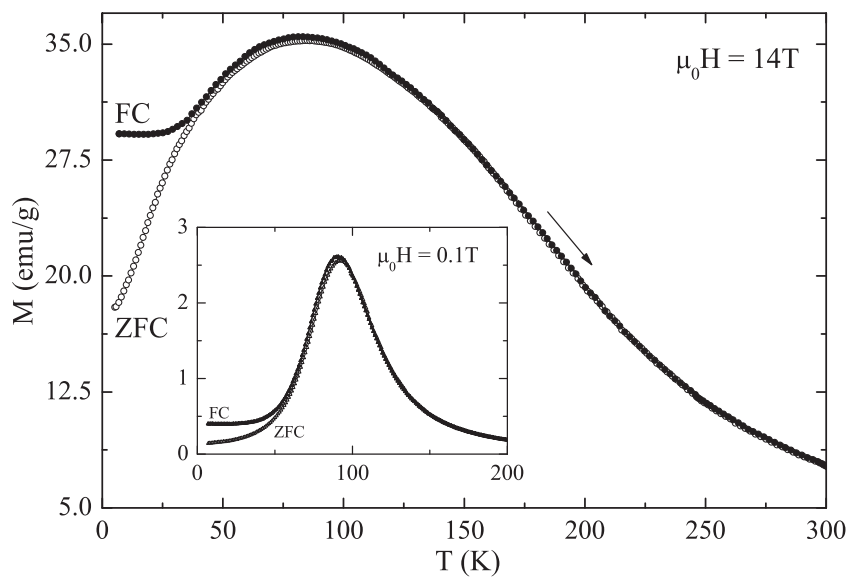

FIG. 4. Magnetization vs. temperature dependencies for 0.3 sample. 


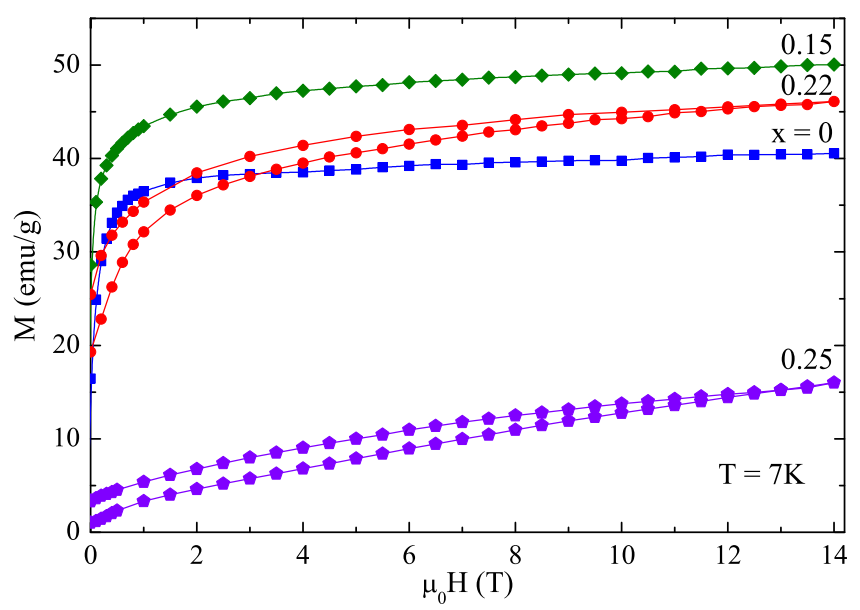

FIG. 5. Magnetization vs. magnetic field dependencies at $7 \mathrm{~K}$ measured after cooling in a field of $0.1 \mathrm{~T}$.

significantly higher than that for $x=0$. The coercive force for $x=0.05$ is around $0.1 \mathrm{~T}$ at $7 \mathrm{~K}$ and increases with iron content increasing $\left(\mathrm{H}_{\mathrm{C}} \approx 0.2 \mathrm{~T} ; x=0.22\right)$. Note that the cobaltites in spin-glass state exhibit much larger coercive force at low temperature. ${ }^{20}$ Magnetic moments per formula unit evaluated from magnetization data are around $1.8 \mu_{\mathrm{B}}$ $(x=0)$ and $2 \mu_{\mathrm{B}}(x=0.15)$. The observed magnetic moments are close to reported data for metallic $\mathrm{La}_{0.5} \mathrm{Ba}_{0.5} \mathrm{CoO}_{3}$ and $\mathrm{LaBaCO}_{2} \mathrm{O}_{6} \cdot{ }^{10,11,21}$ Taking into account the enhancement of the magnetization under small Fe doping, one can suggest that the compositions $0.05 \leq x \leq 0.15$ are predominantly ferromagnetic. However, nonferromagnetic clusters embedded in ferromagnetic matrix are not excluded. The increasing iron concentration above $x=0.23$ leads to strong drop of the magnetization, thus, indicating a collapse of the long-range ferromagnetic ordering. The isotherms of the magnetization vs. magnetic field for $x=0.3$ composition are presented in Fig. 6. The measurements were taken after cooling in a field of $3 \mathrm{~T}$ in order to exclude effects associated with remnant magnetization. One can see that magnetic field induces magnetic hysteresis at the temperature below $30 \mathrm{~K}$. Furthermore, the magnetic susceptibility slightly increases with increasing magnetic field. This type of behavior can be associated with

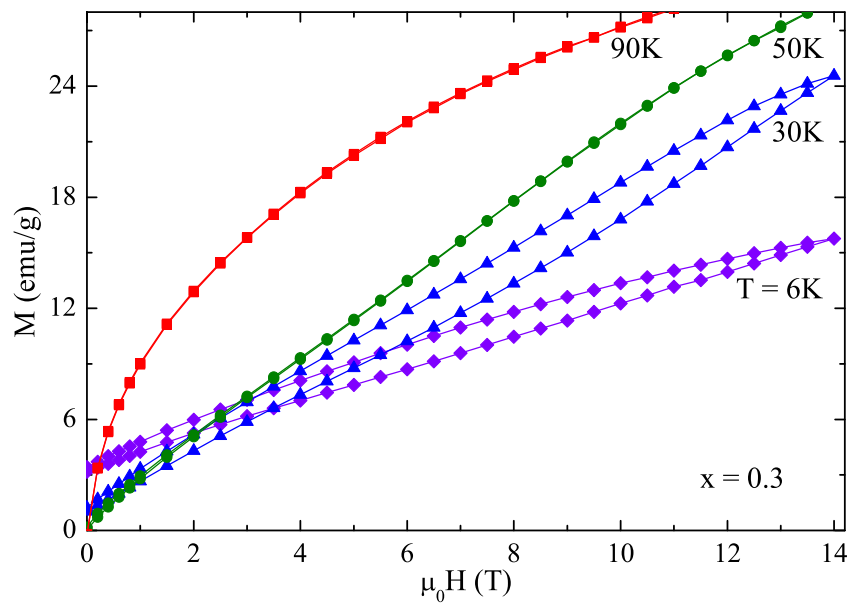

FIG. 6. Magnetization vs. magnetic field dependencies of $x=0.3$ sample measured on heating after cooling in a field of $3 \mathrm{~T}$. metamagnetic phase transition. Magnetic hysteresis disappears at $50 \mathrm{~K}$ and the magnetization vs. field dependence becomes nearly linear. Magnetization vs. field curves above $70 \mathrm{~K}$ are typical of superparamagnets. Hysteretic behavior in strong fields and anomalous decreasing magnetization at low temperature can be ascribed to antiferromagnet-ferromagnet and concomitant spin state transitions induced by magnetic field and temperature, respectively. Note that antiferromagnet-ferromagnet transition induced by magnetic field is irreversible in part below $20 \mathrm{~K}$ because magnetic hysteresis is smaller in second cycle of measurements.

The magnetization behavior of the $\mathrm{La}_{1-y} \mathrm{Ba}_{y} \mathrm{CoO}_{3-\delta}$ $(y>0.5)$ series is displayed in Fig. 7. The increasing of the barium content strongly suppresses the ferromagnetic state. The magnetization decreases as temperature lowers. Taking into account NPD data (Fig. 2), one can conclude that $\mathrm{La}_{0.5} \mathrm{Ba}_{0.5} \mathrm{CoO}_{3-\delta}$ composition is nearly concentration ferromagnet-antiferromagnet transition and therefore can contain antiferromagnetic clusters.

\section{Resistivity and magnetoresistivity}

The Fe-doping stabilizes insulating state. The composition $x=0$ above $150 \mathrm{~K}$ exhibits temperature dependence of resistivity typical of metals. ${ }^{13}$ About $\mathrm{T}_{\mathrm{C}} \approx 170 \mathrm{~K}$, resistivity vs. temperature curve has a break associated with magnetic ordering. Below $150 \mathrm{~K}$, the resistivity increases as temperature

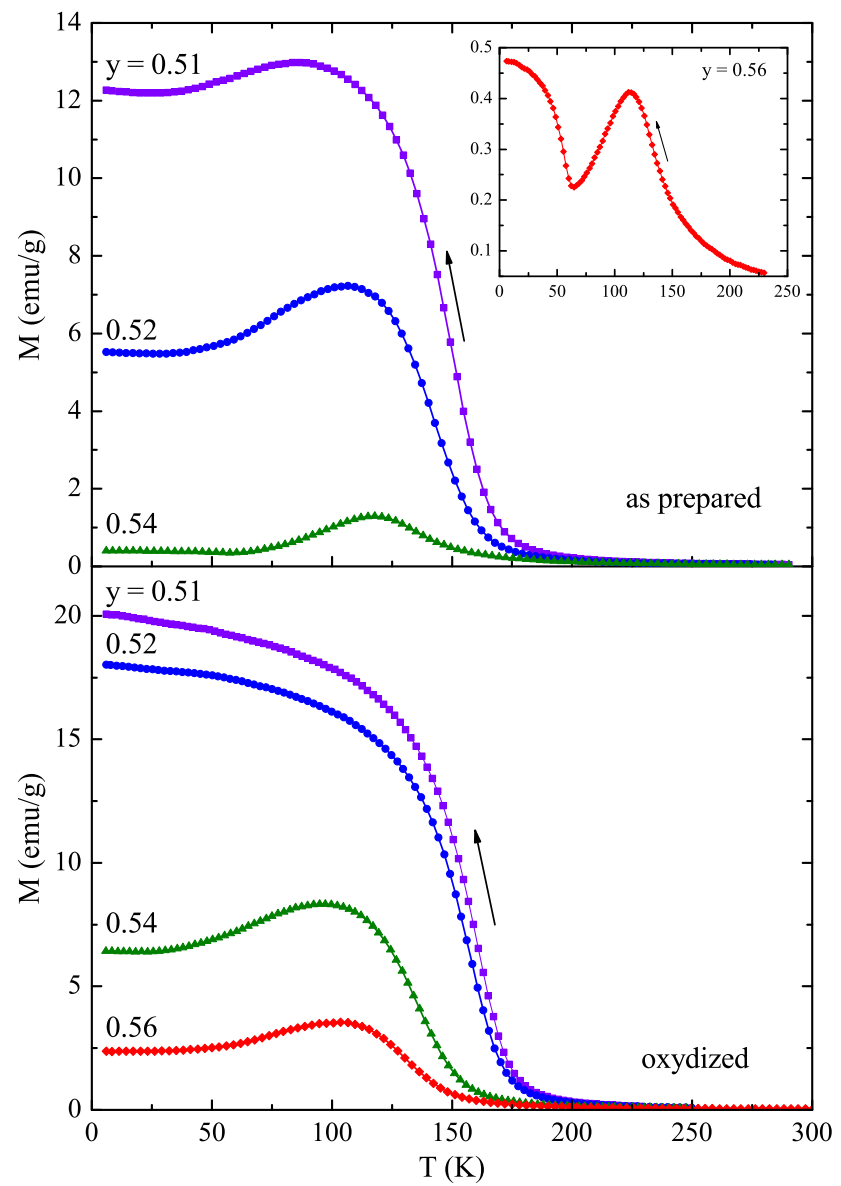

FIG. 7. Magnetization vs. temperature dependencies of the $\mathrm{La}_{1-y} \mathrm{Ba}_{y} \mathrm{CoO}_{3-\delta}$ (as prepared and oxidized) measured in a field of $0.1 \mathrm{~T}$ upon cooling. 


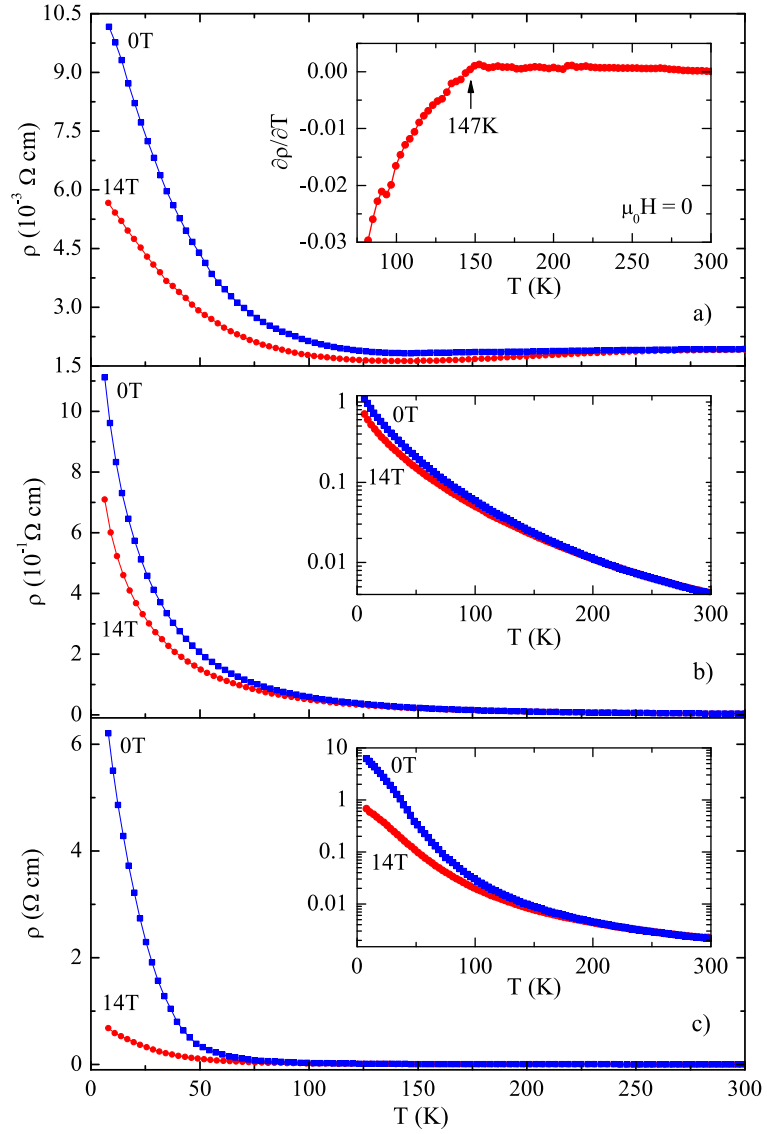

FIG. 8. Resistivity vs. temperature dependencies for $x=0.05$ (a), $x=0.1$ (b), and $x=0.2$ (c) compositions. The insets show $\partial \rho / \partial T$ in vicinity of the Curie point (a) and resistivity behavior in a logarithmic scale (b) and (c).

lowers similar to semiconductors. The temperature dependencies of the resistivity for the composition $x=0.05$ in different magnetic fields are shown in Fig. 8(a). One can see that the metallic behavior transforms in semiconducting one near the Curie point, at that the magnetically ordered phase is weakly insulating (inset to Fig. 8(a)). The dependence $\partial \rho / \partial T$ clearly shows anomaly at Curie point (inset Fig. 8(a)). The compositions with higher Fe ions content are insulating in the whole investigated temperature interval $5-320 \mathrm{~K}$. The $x=0.1$ sample exhibits resistivity by two orders of magnitude larger than the metallic $x=0.05$ sample. Magnetic ordering does not lead to clear anomaly in the resistivity for samples $0.1 \leq x \leq 0.2$ near the Curie point (Figs. 8(b) and 8(c)). However, the anomalous resistivity behavior occurs at low temperature for $x=0.2$ sample (inset to Fig. $8(\mathrm{c})$ ) where the drop of the magnetization was observed (Fig. 3).

Magnetoresistivity dependence on temperature is not monotonous for the composition $x=0$ : near the Curie point the maximum is observed, however, below $130 \mathrm{~K}$ the magnetoresistivity starts to increase again by lowering temperature. ${ }^{13}$ Fig. 9 demonstrates the magnetoresistivity dependent on magnetic field measured for $x=0.2$ at $7 \mathrm{~K}$. The magnetoresistance is defined as $M R=\{[\rho(H)-\rho(0)] / \rho(H)\} \cdot 100 \%$, where $\rho(0)$ and $\rho(H)$ are the resistivity values at zero field and applied magnetic field, respectively. One can see that the dependence is practically linear as the magnitude of magnetic field decreases. In large fields, there is not observed any tend-

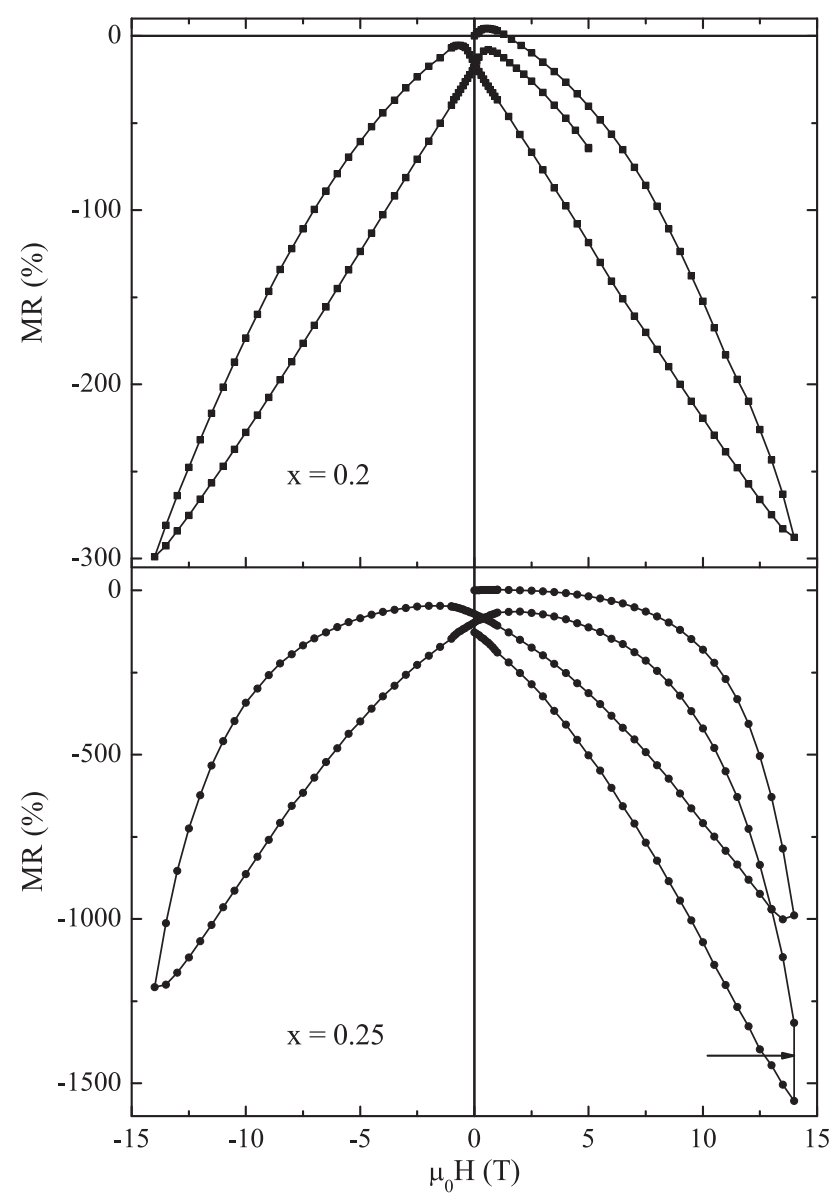

FIG. 9. Magnetoresistance vs. field dependencies of $x=0.2$ and $x=0.25$ samples. The measurements are taken after zero field cooling. The arrow indicates an exposition during $5 \mathrm{~min}$ in a field of $14 \mathrm{~T}$.

ency to saturation. Besides, a large magnetic hysteresis is evident at low temperature. It can be noted that magnetoresistance has positive and negative components. The positive component is pronounced at low temperature and low field regime (Fig. 9). This component is not related directly to magnetic moments reorientation of the ferromagnetic matrix because coercive field of $x=0.2$ sample is less than $0.2 \mathrm{~T}$. The magnetoresistance hysteresis in strong magnetic fields (observed in all the $0.05 \leq x \leq 0.25$ compositions at $7 \mathrm{~K}$ ) seems to be result of the competition between positive and negative components. The transition from ferromagnetic state into antiferromagnetic one $(x=0.25)$ is accompanied by strong enhancement of the magnetoresistance (Fig. 9). Sample with $x=0.25$ is relaxed forward to lower resistivity in a field of $14 \mathrm{~T}$ (Fig. 9). Magnetoresistance and magnetic hysteresis strongly decrease as temperature rises. The positive component disappears around $100 \mathrm{~K}$. Magnetoresistivity increases rather sharply as Fe content increases excluding the $x=0.1$ compound. This sample shows magnetoresistance similar to $x=0.05$ composition.

The magnetoresistance depends on magnetic prehistory. The effect measured after cooling in a field differs from that after cooling in zero field (Figs. 9 and 10). In the compositions $x=0.2$ and $x=0.3$, the cooling in an external magnetic field of $14 \mathrm{~T}$ leads to lowering electroresistivity almost by 1 and 3 orders of magnitude, respectively (Fig. 10). To our knowledge, there are the largest reported values of the 


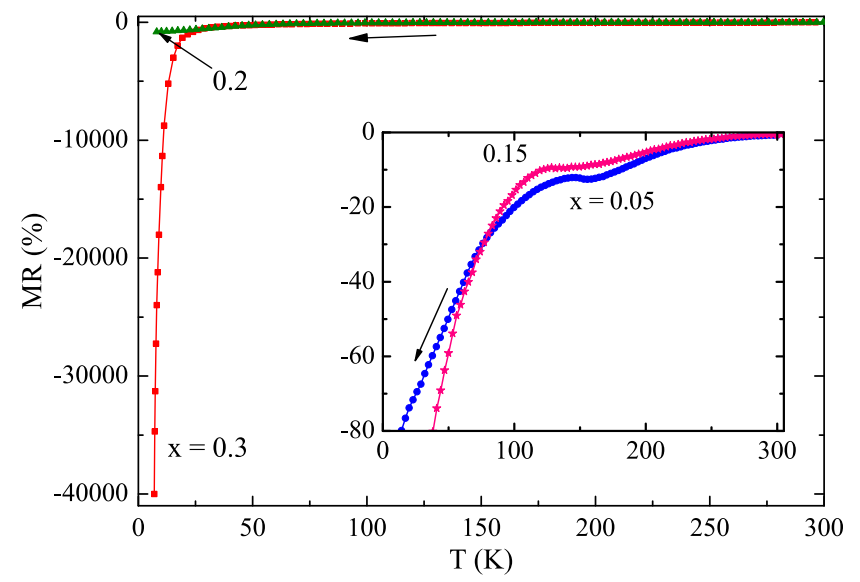

FIG. 10. Magnetoresistance vs. temperature dependencies obtained as difference of the resistivity measured in zero field and field of $14 \mathrm{~T}$ upon cooling.

magnetoresistance in the cobaltites. Note that magnetoresistance in the metallic paramagnetic phase of the $x=0.05$ sample exhibits weakly pronounced maximum near the Curie point (inset Fig. 10).

The increasing barium content above $y=0.5$ in $\mathrm{La}_{1-y} \mathrm{Ba}_{y} \mathrm{CoO}_{3-\delta}$ series leads to insulating behavior below $300 \mathrm{~K}$ (Fig. 11). Nearly $\mathrm{T} \approx 150 \mathrm{~K}$, the $\rho(T)$ shows anomaly: below this temperature, the resistivity rises much faster than at $T>150 \mathrm{~K}$. Note that metal-semiconductor transition in $y=0.5$ composition occurs at $150 \mathrm{~K}$ too (Fig. 11). The magnetoresistance rises with increasing barium content reaching maximum in $y=0.54$ composition (inset Fig. 11).

It is known that the low field intergrain tunneling magnetoresistance is an indicator of spin polarization of the charge carriers. $^{22}$ However, we did not observe any corresponding contribution into magnetoresistance in the low field regime for all the studied metallic and insulating cobaltites. Our findings are in agreement with the data reported in the works. ${ }^{11,15,23}$

\section{DISCUSSION}

The cobalt ions in the high, low, and intermediate spin states have different electronic configuration and therefore chemical bond should be noticeable different. Really, a con-

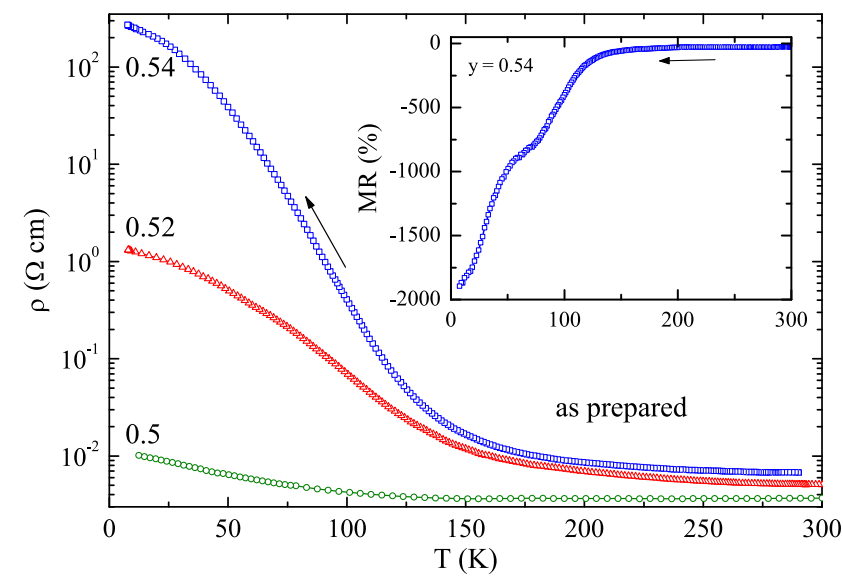

FIG. 11. Resistivity vs. temperature dependencies of the $\mathrm{La}_{1-y} \mathrm{Ba}_{y} \mathrm{CoO}_{3-\delta}$ as prepared compositions. The inset shows magnetoresistance ratio vs. temperature. centration transition from low spin state into intermediate one in lightly doped $\mathrm{La}_{1-y} \mathrm{Sr}_{y} \mathrm{CoO}_{3}$ system is accompanied by phase separation. ${ }^{20}$ The macroscopic structural phase separation into antiferromagnetic and ferromagnetic phases has been observed in anion-deficient $\mathrm{La}_{0.5} \mathrm{Ba}_{0.5} \mathrm{CoO}_{3-\delta .}{ }^{13,24}$ The phase separation occurs concomitantly with the onset of antiferromagnetic ordering $\left(T_{N} \sim 150 \mathrm{~K}\right)$. This effect has been treated in terms of spin state transition induced by stabilization of the antiferromagnetic phase. ${ }^{13,24}$ The antiferromagnetic phase has insignificantly larger unit cell than ferromagnetic one. ${ }^{13,24}$ So, the antiferromagnetic phase was ascribed to a mixed high/low spin state, whereas ferromagnetic phase is in intermediate spin state. ${ }^{24}$

The anomalous broadening of the diffraction peaks observed around $100 \mathrm{~K}$ in the $\mathrm{La}_{0.5} \mathrm{Ba}_{0.5} \mathrm{Co}_{0.75} \mathrm{Fe}_{0.25} \mathrm{O}_{3-\delta}$ composition (Fig. 1) can be explained in terms of spin state transition and phase separation scenario. Apparently, above $150 \mathrm{~K}$, the intermediate spin state of the cobalt ions is stable. The lowering temperature down to $150 \mathrm{~K}$ leads to phase separated state consisting of ferromagnetic and antiferromagnetic phases in different spin states of cobalt ion. The further temperature lowering down to $2 \mathrm{~K}$ stabilizes antiferromagnetic phase and sample becomes more homogeneous. The spin state transition is accompanied by narrowing of the diffraction peaks and increasing of the unit cell volume observed below $100 \mathrm{~K}$. The extremely weak dependence of the unit cell volume on temperature and strains release below $100 \mathrm{~K}$ observed in $\mathrm{La}_{0.44} \mathrm{Ba}_{0.56} \mathrm{CoO}_{3-\delta}$ composition (Fig. 2) is apparently associated with stabilization of the antiferromagnetic phase too. The structural behavior is well correlated with drop of the magnetization (Figs. 3 and 7). Antiferromagnetic and ferromagnetic orderings begin to develop approximately at the same temperature because magnetic ordering is accompanied by increase in resistivity (Fig. $11)$. It is in agreement with the NPD and X-ray data of anion deficient $\mathrm{La}_{0.5} \mathrm{Ba}_{0.5} \mathrm{CoO}_{3-\delta}{ }^{13,24}$ One can conclude that antiferromagnetic phase with mixed high/low spin state of the cobalt ions is insulating (Fig. 11).

The spontaneous magnetization observed in ferromagnetic compositions $\mathrm{La}_{0.5} \mathrm{Ba}_{0.5} \mathrm{Co}_{1-x} \mathrm{Fe}_{x} \mathrm{O}_{3-\delta}(x<0.2)$ is less than expected value $-2.5 \mu_{B}$ for the intermediate spin state of the $\mathrm{Co}^{3+}\left(S=1 ; t_{2 g}^{5} e_{g}^{1}\right)$ and $\mathrm{Co}^{4+}\left(S=3 / 2 ; t_{2 g}^{4} e_{g}^{1}\right)$ ions and corresponds to the data reported for metallic $\mathrm{La}_{0.5} \mathrm{Ba}_{0.5} \mathrm{CoO}_{3}$ and $\mathrm{LaBaCo}_{2} \mathrm{O}_{6} \cdot{ }^{10,11,21}$ However, cobaltites are negative charge transfer materials. ${ }^{25,26}$ There is no change in oxidative state of cobalt ions in $\mathrm{La}_{1-y} \mathrm{Sr}_{y} \mathrm{CoO}_{3}$ up to $y=0.5$ as it was evidenced by XAS. ${ }^{25,26}$ So, the observed magnetic moment $\left(\sim 2 \mu_{B}\right)$ can be explained suggesting intermediate spin state of $\mathrm{Co}^{3+}$ ion $\left(S=1 ; t_{2 g}^{5} e_{g}^{1}\right)$. The contribution from itinerant holes seems to be small because intergranular tunneling magnetoresistance is not observed.

Mössbauer study has shown that iron ions in cobaltites can be in delocalized $\mathrm{Fe}^{3+\alpha}$ and high spin localized $\mathrm{Fe}^{4+}$ $\left(s=2 ; t_{2 g}^{3} e_{g}^{1}\right)$ states. $^{27}$ Apparently, metal-insulator transition in iron doped samples $(x>0.05)$ is accompanied by crossover $\mathrm{Fe}^{3+\alpha}$ into $\mathrm{Fe}^{4+}$ high spin state. It leads to increasing both magnetic moment and Curie point (Fig. 3). Note that the highest Curie point $\left(T_{C}=340 \mathrm{~K}\right)$ is observed in $\mathrm{SrCo}_{0.5-}$ $\mathrm{Fe}_{0.5} \mathrm{O}_{3}{ }^{3}$ Magnetic moment $3 \mu_{B}$ (Ref. 3) can be understood 
suggesting intermediate spin state of $\mathrm{Co}^{3+}$ ion and high spin state of $\mathrm{Fe}^{4+}$ ion. The Curie point of $\mathrm{SrCoO}_{3}$ is $305 \mathrm{~K}$ and measured magnetic moment around $2.5 \mu_{B}{ }^{28}$ The magnitude of magnetic moment formally corresponds to intermediate spin state of $\mathrm{Co}^{3+}$ and $\mathrm{Co}^{4+}$ ions mixed in 1:1 ratio.

However, $\mathrm{Co}^{3+}$ and $\mathrm{Co}^{4+}$ in intermediate spin state and $\mathrm{Fe}^{4+}$ in high spin state are Jahn-Teller ions because they contain only one $e_{g}$ electron. The static long-range Jahn-Teller distortion in cobaltites seems to be unlikely, because they are negative charge transfer materials with strong metalligand hybridization. ${ }^{16}$ In contrast to orbital ordered manganites, ${ }^{16}$ the "insulating" state of the $\mathrm{La}_{0.5} \mathrm{Ba}_{0.5} \mathrm{CoO}_{3}$ is very conductive. $^{10,11}$ Slightly anion-deficient $\mathrm{La}_{0.5} \mathrm{Ba}_{0.5} \mathrm{CoO}_{2.99}$ shows well pronounced metallic behavior up to $50 \mathrm{~K}^{21}$ Moreover, the extended X-ray absorption fine structure (EXAFS) and neutron diffraction studies do not reveal any appreciable local Jahn-Teller distortion in $\mathrm{La}_{1-y} \mathrm{Sr}_{y} \mathrm{CoO}_{3}{ }^{29}$ One can suggest that magnetostructural distortions in the $\mathrm{La}_{1-y} \mathrm{Ba}_{y} \mathrm{CoO}_{3-\delta}$ cobaltites could be associated with stabilization of the ferromagnetic state by strains arising from short-range ordering of $\mathrm{Ba}$ and $\mathrm{La}$ ions and antiferromagnetic clusters embedded in ferromagnetic matrix. The magnetostructural distortions are most pronounced in the $y=0.25$ and $y=0.5$ compositions of the $\mathrm{La}_{1-y} \mathrm{Ba}_{y} \mathrm{CoO}_{3} \cdot{ }^{7,10,11}$ Apparently, there is a tendency to short-range ordering of $\mathrm{La}$ and $\mathrm{Ba}$ ions having very different ionic radii. Really, the cubic at room temperature $\mathrm{La}_{0.5} \mathrm{Ba}_{0.5} \mathrm{CoO}_{3}$ has been obtained as nanoscale ordered composition. ${ }^{21}$ Besides tetragonal distortions stabilize the intermediate spin state. In fact, $\mathrm{LaCoO}_{3}$ tetragonal films have ferromagnetic component with the Curie point up to $90 \mathrm{~K}$ and exhibit insulating character. ${ }^{30}$ However, rhombohedral distortion destroys ferromagnetic component. ${ }^{30,31}$ Ferromagnetism of these tetragonal films is probably associated with mixed intermediate/low spin state as a measured magnetic moment is small $\sim 0.7 \mu_{\mathrm{B}} / \mathrm{Co}^{3+} .30,31$

The large unit cell volume of the $\mathrm{La}_{0.5} \mathrm{Ba}_{0.5} \mathrm{Co}_{1-x} \mathrm{Fe}_{x} \mathrm{O}_{3}$ series is in agreement with the concurrence between high and intermediate spin states. Indeed, an applied pressure stabilizes ferromagnetic state in $\mathrm{La}_{0.5} \mathrm{Ba}_{0.5} \mathrm{CoO}_{3-\delta}$ (Ref. 32) and $\mathrm{La}_{0.5} \mathrm{Ba}_{0.5} \mathrm{Co}_{0.9} \mathrm{Ti}_{0.1} \mathrm{O}_{3} .{ }^{14} \mathrm{NMR}$ data for antiferromagnetic cobaltites are in agreement with the coexisting of cobalt ions in low and high spins states. ${ }^{33}$ The contribution of high spin configuration increases with increasing of the unit cell volume. For example, in $\mathrm{Sr}_{2} \mathrm{Co}_{2} \mathrm{O}_{5}$, the measured magnetic moment for $\mathrm{Co}$ in octahedral positions is slightly larger than $3 \mu_{\mathrm{B}}{ }^{34}$ The high spin electronic configuration produces very strong antiferromagnetic superexchange interactions. In fact, magnetic moment of $\mathrm{LaCoO}_{3}$ is not saturated even in a magnetic field of $500 \mathrm{~T} .{ }^{35}$ Really, both $\mathrm{Fe}^{3+}$ and $\mathrm{Co}^{3+}$ in high spin states have two unpaired $e_{g}$-electrons. The Neel point of $\mathrm{LaFeO}_{3}$ is $750 \mathrm{~K}$. However, $d$ - $p$ hybridization is larger in cobaltites.

The behavior of the resistivity observed in $x=0.05$ sample (Fig. 8(a)) is very similar to reported earlier for stoichiometric $\mathrm{La}_{0.5} \mathrm{Ba}_{0.5} \mathrm{CoO}_{3}$ at temperature below $140 \mathrm{~K} .{ }^{11}$ Sample $x=0.05$ is well metallic in paramagnetic state. Samples with $x \geq 0.1$ are well insulating in both paramagnetic and ferromagnetic phases. However, concentration metalinsulator transition practically does not affect the Curie point. Similar result has been received for low-doped $\mathrm{La}_{1-y} \mathrm{Ba}_{y} \mathrm{CoO}_{3-\delta}$ compositions. ${ }^{7}$ This type of behavior can indicate similar origin of the ferromagnetism for metallic and insulating cobaltites. Apparently, the compounds with $x \geq 0.1$ are negative charge transfer insulators as it was found for layered perovskites $\mathrm{Sr}_{3} \mathrm{Fe}_{2}{ }^{4+} \mathrm{O}_{7}$ and $\mathrm{Sr}_{2} \mathrm{Fe}^{4+} \mathrm{O}_{4}$ by $\mathrm{X}$-ray photoelectron spectroscopy. ${ }^{36}$ It means that the band gap is of the $p-p$ type. $^{36}$

The double exchange and itinerant magnetism are not responsible for ferromagnetism of the metallic cobaltites because insulating cobaltites demonstrate similar ferromagnetic behavior and there is not any intergrain tunneling magnetoresistance (Fig. 9). It assumes a week spin polarization of the charge carriers. The situation with magnetic interactions in ferromagnetic metallic cobaltites seems to be similar in some aspects to that in the insulating ferromagnetic phase of $\mathrm{LaCoO}_{3}$. Really, the surface ferromagnetism has been revealed in $\mathrm{LaCoO}_{3}$ and ascribed to $\mathrm{Co}^{3+}$ ions on the surface being in the intermediate spin state. ${ }^{37}$ Goodenough interpreted the surface ferromagnetism of $\mathrm{LaCoO}_{3}$ using the model of superexchange interactions via oxygen ions taking into account the magneto-vibrational coupling. ${ }^{37}$

The large magnetoresistance in the low doped cobaltites has been treated on the analogy of granular giant magnetoresistance assuming the presence of nanoscale ferromagnetic particles in a metallic or insulating matrix. ${ }^{20}$ However, $\mathrm{La}_{0.5} \mathrm{Ba}_{0.5} \mathrm{Co}_{1-x} \mathrm{Fe}_{x} \mathrm{O}_{3-\delta}(x \leq 0.23)$ are predominantly ferromagnetic. The magnetoresistance is the most pronounced in vicinity of critical iron concentration related to transition from long-range ferromagnetic order to antiferromagnetic state (Fig. 9). We suppose the large negative magnetoresistance at low temperatures (where intermediate spin state is destabilized) to be associated with antiferromagnet-ferromagnet transition induced by external magnetic field. Our suggestion is in agreement with the results published earlier. ${ }^{8,38}$ The strong suppression of the antiferromagnetic phase by magnetic field was detected in $\mathrm{La}_{0.82} \mathrm{Ba}_{0.18} \mathrm{CoO}_{3-\delta}$ using NPD study in magnetic field. ${ }^{8}$ A huge anisotropic magnetostriction (corresponding to a tetragonal distortion) was observed in initially rhombohedral $\mathrm{La}_{0.7} \mathrm{Sr}_{0.3} \mathrm{CoO}_{3}$ and ascribed to spin state transition induced by magnetic field. ${ }^{38}$ Tetragonal distortion stabilizes the intermediate spin state. Magnetoresistance at low temperature has positive and negative components (Fig. 9). Apparently, positive component can be ascribed to splitting of $e_{g}$-band, whereas the negative component can arise from decreasing of the gap between $e_{g}$ and $t_{2 g}$ levels. Cooling in a strong magnetic field supports tetragonal distortion thus leading to much larger magnetoresistance in comparison with that measured after zero field cooling (Figs. 9 and 10). The antiferromagnetic phase is characterized by much larger resistivity than ferromagnetic one (Fig. 11). Apparently, both antiferromagnetic and ferromagnetic phases are negative charge transfer insulators. It means that cobalt oxidative state is close to $3+, p-d$ charge transfer integral is larger than width of $2 p$ band and insulating gap is $p-p$ type ${ }^{36}$ However, the insulating gap in the antiferromagnetic phase is much larger due to high and low spin electronic configurations of $\mathrm{Co}^{3+}$ ions. It leads to strong enhancement of $p$ - $d$ charge transfer integral. 
Note that anion-deficient $\operatorname{Pr}_{0.5} \mathrm{Ca}_{0.5} \mathrm{CoO}_{3-\delta}$ exhibits macroscopic structural phase separation into ferromagnetic and nonmagnetic phases. ${ }^{39}$ In this case, unit cell volume is too small to stabilize $\mathrm{Co}^{3+}$ ion in high spin state. Spin state transition in "stoichiometric" $\mathrm{Pr}_{0.5} \mathrm{Ca}_{0.5} \mathrm{CoO}_{3}$ is assisted by partial excitation of $\mathrm{Pr}^{3+}[15( \pm 5) \%]$ into $\mathrm{Pr}^{4+}$ ions. ${ }^{40}$ Apparently, it is necessary for transition into regime of negative charge transfer insulator. In $\mathrm{La}_{1-y} \mathrm{Ba}_{y} \mathrm{CoO}_{3-\delta}$ series, the width of $2 p$ band is regulated by anion deficiencies.

\section{CONCLUSIONS}

To summarize, we have shown that the $\mathrm{La}_{0.5} \mathrm{Ba}_{0.5} \mathrm{Co}_{1-x} \mathrm{Fe}_{x} \mathrm{O}_{3-\delta}$ compositions are predominantly ferromagnetic at $x \leq 0.23$ and predominantly antiferromagnetic at $x \geq 0.25$. The insulating composition $(x=0.1)$ shows larger magnetization and Curie point than metallic one $(x=0.05)$. The enhancement of the barium content in $\mathrm{La}_{1-y} \mathrm{Ba}_{y} \mathrm{CoO}_{3-\delta}$ series above $y>0.5$ gradually converts ferromagnetic state into antiferromagnetic one too. The antiferromagnetic ordering in $\mathrm{La}_{1-y} \mathrm{Ba}_{y} \mathrm{CoO}_{3-\delta}$ series leads to strong enhancement of the resistivity arising from concomitant spin state transition. The ferromagnet-antiferromagnet transition is accompanied by spin state transition. All the compositions exhibit a large magnetoresistance reaching maximum near the border of the concentration ferromagnetantiferromagnet transition. The large magnetoresistance is associated with antiferromagnet-ferromagnet transition induced by external magnetic field. It is suggested that both semiconducting ferromagnetic and insulating antiferromagnetic phases are negative charge transfer materials. Magnetism is governed by superexchange interactions via oxygen.

\section{ACKNOWLEDGMENTS}

The authors are grateful to J. B. Goodenough, B. Raveau, C. Leighton, and I. Fita for discussions. This work was supported by the Belarusian Republican Foundation for Fundamental Research (Project F12R-057) and partly based on experiments performed at the Swiss spallation neutron source SINQ, Paul Scherrer Institute, Villigen, Switzerland.

${ }^{1}$ P. M. Raccah and J. B. Goodenough, Phys. Rev. 155, 932 (1967).

${ }^{2}$ P. G. Radaelli and S.-W. Cheong, Phys. Rev. B 66, 094408 (2002).

${ }^{3}$ C. Yin, Q. Liu, R. Decourt, M. Pollet, E. Gaudin, and O. Toulemonde, J. Solid State Chem. 184, 3228 (2011).

${ }^{4}$ C. He, H. Zheng, J. F. Mitchell, M. L. Foo, R. J. Cava, and C. Leighton, Appl. Phys. Lett. 94, 102514 (2009).

${ }^{5}$ J.-Q. Yan, J.-S. Zhou, and J. B. Goodenough, Phys. Rev. B 69, 134409 (2004).

${ }^{6}$ J. Wu and C. Leighton, Phys. Rev. B 67, 174408 (2003).

${ }^{7}$ P. Tong, J. Yu, Q. Huang, K. Yamada, and D. Louca, Phys. Rev. Lett. 106, 156407 (2011)

${ }^{8}$ P. Tong, Q. Huang, M. Kofu, M. C. Lehman, J. Yu, and D. Louca, J. Phys.: Conf. Ser. 251, 012015 (2010).
${ }^{9}$ A. P. Sazonov, I. O. Troyanchuk, H. Gamari-Seale, V. V. Sikolenko, K. L. Stefanopoulos, G. K. Nicolaides, and Y. K. Atanassova, J. Phys.: Condens. Matter 21, 156004 (2009).

${ }^{10}$ F. Fauth, E. Suard, and V. Caignaert, Phys. Rev. B 65, 060401(R) (2001).

${ }^{11}$ T. Nakajima, M. Ichihara, and Y. Ueda, J. Phys. Soc. Jpn. 74, 1572 (2005).

${ }^{12}$ I. O. Troyanchuk, A. N. Chobot, N. V. Tereshko, D. V. Karpinskii, V. Efimov, V. Sikolenko, and P. Henry, JETP 112, 837 (2011).

${ }^{13}$ I. O. Troyanchuk, D. V. Karpinsky, M. V. Bushinsky, V. Sikolenko, V. Efimov, A. Cervellino, and B. Raveau, J. Appl. Phys. 112, 013916 (2012).

${ }^{14}$ S. Zhang, Y. Zhang, L. Pi, and S. Tan, J. Appl. Phys. 109, 07E131 (2011).

${ }^{15}$ I. O. Troyanchuk, M. V. Bushinskii, D. V. Karpinsky, V. M. Dobryanskii, V. V. Sikolenko, and A. M. Balagurov, JETP Lett. 89, 319 (2009).

${ }^{16}$ J. B. Goodenough and J. S. Zhou, Structure and Bonding (Springer, New York, 2001), pp. 17-113.

${ }^{17}$ T. Roisnel and J. Rodríquez-Carvajal, J. Mater. Sci. Forum 378-381, 118 (2001).

${ }^{18}$ N. Hayashi, T. Yamamoto, H. Kageyama, M. Nishi, Y. Watanabe, T. Kawakami, Y. Matsushita, A. Fujimori, and M. Takano, Angew. Chem., Int. Ed. 50, 12547 (2011).

${ }^{19}$ A. S. Wills, Physica B 276-278, 680 (2000).

${ }^{20}$ J. Wu, J. W. Lynn, C. J. Glinka, J. Burley, H. Zheng, J. F. Mitchell, and C. Leighton, Phys. Rev. Lett. 94, 037201 (2005).

${ }^{21}$ E.-L. Rautama, P. Boullay, A. K. Kundu, V. Caignaert, V. Pralong, M. Karppinen, and B. Raveau, Chem. Mater. 20, 2742 (2008).

${ }^{22}$ M. Ziese, Rep. Prog. Phys. 65, 143 (2002).

${ }^{23}$ A. Maignan, C. Martin, M. Hervieu, and B. Raveau, J. Magn. Magn. Mater. 211, 173 (2000)

${ }^{24}$ I. O. Troyanchuk, D. V. Karpinsky, M. V. Bushinsky, V. Sikolenko, V. Efimov, and A. Cervellino, JETP Lett. 93, 139 (2011).

${ }^{25}$ J. L. Hueso, J. P. Holgado, R. Pereñiguez, S. Mun, M. Salmeron, and A. Caballero, J. Solid State Chem. 183, 27 (2010).

${ }^{26}$ O. Haas, C. Ludwig, U. Bergmann, R. Singh, A. Braun, and T. Graule, J. Solid State Chem. 184, 3163 (2011).

${ }^{27}$ A. Maignan, C. Martin, N. Nguyen, and B. Raveau, Solid State Sci. 3, 57 (2001).

${ }^{28}$ Y. Long, Y. Kaneko, S. Ishiwata, Y. Taguchi, and Y. Tokura, J. Phys.: Condens. Matter 23, 245601 (2011).

${ }^{29}$ N. Sundaram, Y. Jiang, I. E. Anderson, D. P. Belanger, C. H. Booth, F. Bridges, J. F. Mitchell, T. Proffen, and H. Zheng, Phys. Rev. Lett. 102, 026401 (2009).

${ }^{30}$ S. Park, P. Ryan, E. Karapetrova, J. W. Kim, J. X. Ma, J. Shi, J. W. Freeland, and W. Wu, Appl. Phys. Lett. 95, 072508 (2009).

${ }^{31}$ D. Fuchs, L. Dieterle, E. Arac, R. Eder, P. Adelmann, V. Eyert, T. Kopp, R. Schneider, D. Gerthsen, and H. v. Löhneysen, Phys. Rev. B 79, 024424 (2009)

${ }^{32}$ I. Fita, R. Szymczak, R. Puzniak, A. Wisniewski, I. O. Troyanchuk, D. V. Karpinsky, V. Markovich, and H. Szymczak, Phys. Rev. B 83, 064414 (2011).

${ }^{33}$ M. Itoh, Y. Nawata, T. Kiyama, D. Akahoshi, N. Fujiwara, and Y. Ueda, Physica B 329-333, 751 (2003).

${ }^{34}$ A. Muñoz, C. de la Calle, J. A. Alonso, P. M. Botta, V. Pardo, D. Baldomir, and J. Rivas, Phys. Rev. B 78, 054404 (2008).

${ }^{35}$ V. V. Platonov, Y. B. Kudasov, M. P. Monakhov, and O. M. Tatsenko, Phys. Solid State 54, 279 (2012).

${ }^{36}$ M. Abbate, H. Ascolani, F. Prado, and A. Caneiro, Solid State Commun. 129, 113 (2004).

${ }^{37}$ J.-Q. Yan, J.-S. Zhou, and J. B. Goodenough, Phys. Rev. B 70, 014402 (2004).

${ }^{38}$ M. R. Ibarra, R. Mahendiran, C. Marquina, B. García-Landa, and J. Blasco, Phys. Rev. B 57, R3217 (1998).

${ }^{39}$ A. Chichev, M. Dlouhá, S. Vratislav, J. Hejtmánek, Z. Jirák, K. Kniížek, and M. Maryško, Z. Kristallogr. Suppl. 26, 435 (2007).

${ }^{40}$ J. Herrero-Martín, J. L. García-Muñoz, S. Valencia, C. Frontera, J. Blasco, A. J. Barón-González, G. Subías, R. Abrudan, F. Radu, E. Dudzik, and R. Feyerherm, Phys. Rev. B 84, 115131 (2011). 